\title{
INTERLEUKIN-8 VALUES IN PEDIATRIC ONCOLOGY PATIENTS WITH FEBRILE NEUTROPENIA AND BLOODSTREAM INFECTIONS
}

\author{
V. Urbonas ${ }^{1}$, A. Eidukaite $^{2}$, I. Tamuliene ${ }^{3}$ \\ ${ }^{1}$ Immunology Dept, Center of Innovative Medicine, ${ }^{2}$ Center of Innovative Medicine, National Research \\ Institute, ${ }^{3}$ Vilnius University Children's Hospital, Vilnius, Lithuania
}

Background and aims: Upon bacterial stimulation, leukocytic cells and non-leukocytic somatic cells produce variety of cytokines which regulate immune response, inflammatory and hematopoiesis processes. One of these cytokines - interleukin-8 (IL-8) - takes part in leukocytic cells homing and chemotaxis, wound healing, angiogenesis, tumor development.

The aim of this study was to evaluate and compare of values of IL-8 in oncohematologic children with febrile neutropenia (FN) and bloodstream infections.

Methods: A total of 16 febrile neutropenic episodes in 16 children were studied. Serum samples were collected on day 1 and day 2. Microbiological evaluation and IL-8 determination of the samples were performed. Comparison of IL-8 values was performed using Mann-Whitney non-parametric statistical test.

Results: Gram-negative infection was detected in 11 children, whereas Gram-positive infection - in 5 children. Patients with Gram-negative infection have IL-8 values of 3,9 - 4,3 times higher than patients with Gram-positive infection (see Table 1).

Conclusions: These data showed a distinct innate immune response to different types of bacteria in oncohematologic children with FN. IL-8 could be used as a additional diagnostic tool in children with FN and malignancies for sepsis or bacteremia determination on the first days of fever. In the meantime, validation of IL-8 in various diagnostic settings is required. 